Article

\title{
Bioremediation of Landoltia punctata to Microcystis aeruginosa Contaminated Waters
}

\author{
Shi Li ${ }^{\dagger}$, Sixiu Le ${ }^{\dagger}$, Guolin Li, Mei Luo, Rui Wang * and Yun Zhao *® \\ Key Laboratory of Bio-Resource and Eco-Environment of Ministry of Education, College of Life Sciences, \\ Sichuan University, No. 24 South Section 1, Yihuan Road, Chengdu 610065, China; lishi9402@163.com (S.L.); \\ lesixiu@163.com (S.L.); 1g1231314@sina.com (G.L.); luomei@163.com (M.L.) \\ * Correspondence: wangray1987@scu.edu.cn (R.W.); zhaoyun@scu.edu.cn (Y.Z.); \\ Tel.: +86-28-85418776 (R.W. \& Y.Z.); Fax: +86-28-85412485 (R.W. \& Y.Z.) \\ + These authors contributed equally to this work.
}

Received: 19 May 2020; Accepted: 17 June 2020; Published: 21 June 2020

check for

\begin{abstract}
Microcystis aeruginosa is one of the dominant algae in the "phytoplankton bloom" phenomenon. A high density of microcystins (MCs) are produced when algae have explosive growth, which can damage the water environment and pose a great threat to aquatic animals, plants, and human health. Duckweed (Landoltia punctata) is a morphologically highly degraded flowering plant with a short growth cycle and wide environmental adaptability. Importantly, duckweed can grow in eutrophic water and has great potential in water remediation. The present study aims to analyze the physiological and biochemical changes of L. punctata when co-culturing with $M$. aeruginosa in the laboratory. Our results showed that all the biomass, chlorophyll content, antioxidant enzyme activities, and amylase activity of L. punctata increased in $2 \times 10^{8}$ cells/L and $4 \times 10^{8}$ cells/L for M. aeruginosa, and also significantly reduced in $1.6 \times 10^{9}$ cells/L for M. aeruginosa, while cytotoxic substance (malondialdehyde (MDA)) content showed a completely opposite trend. After co-culturing, it was found that the MC content in L. punctata reduced to $138.87 \mathrm{~g} / \mathrm{g}$, and the MC removal rate was $29.48 \%$. These results indicate that L. punctata can grow normally in high-density M. aeruginosa, which paves the way for L. punctata's bioremediation of water polluted by M. aeruginosa.
\end{abstract}

Keywords: Landoltia punctata; Microcystis aeruginosa; stress response; antioxidant enzymes; water remediation

\section{Introduction}

Over the years, the intensification of surface water eutrophication has become the most important problem of water quality worldwide [1]. One of the numerous consequences of this process is the increase of algae in their frequency and biomass. Among bloom-forming algae, M. aeruginosa is one of the most intensively studied species [2]. During the growth process of M. aeruginosa, a large amount of microcystins (MCs) is generated, which poses a serious threat to the ecological environment. Various methods are used to counteract algae blooms and their consequences, including physical repair, chemical control, and bioremediation. Due to the inexpensive and high-efficiency features on water remediation, the chemical method is prevalently utilized in practice. However, it has certain limitations and easily causes secondary pollution to the water environment. Hence, phytoremediation, a cost-effective and environmentally friendly method, is of great interest $[3,4]$. As a new type of environmental and energy plant, duckweed has the advantages of high biomass and significant removal of nitrogen and phosphorus from water bodies. It has the characteristics of low energy consumption, low cost, and environmental friendliness in the treatment process [5], thus proving to be a good application prospect in environmental pollution control [6]. 
"Phytoplankton bloom" often occurs in eutrophic water bodies, of which the main reason is the explosive growth of phytoplankton, especially algae. M. aeruginosa, a member of microcystis, is one of the dominant algae species in "phytoplankton bloom", during which a lot of MCs are produced [7]. MCs is a class of cyclo-heptapeptide compounds with strong toxicity to liver function [8], and with their unique structure, they show high resistance to adverse conditions (e.g., $\mathrm{pH}$, high temperature) $[9,10]$. Consequently, the removal of MCs becomes an immediate challenge ahead [11]. Studies have shown that the content of MCs deceased after co-culturing with duckweed in wastewater [6]. More importantly, the growth of duckweed was not compromised at all [12], which made the co-culturing of duckweed and $M$. aeruginosa a potential approach to remove MCs, and showed great research significance.

In the present work, L. punctata and M. aeruginosa were subjected for co-culturing in eutrophic water for 15 days. The biomass, chlorophyll content, antioxidant enzyme activity, malondialdehyde (MDA) content, amylase activity, and starch content of L. punctata were measured after co-cultivation. In addition, the removal efficiency of MCs and the remediation efficiency of wastewater were also estimated. Our results show that L. punctate can significantly reduce the content of MCs, total phosphorus (TP), and total nitrogen (TN) water polluted by M. aeruginosa, which could provide experimental basis for water remediation through the application of L. punctate.

\section{Materials and Methods}

\subsection{Experimental Organisms}

M. aeruginosa (FACHB-930) was purchased from the freshwater algae species bank of Wuhan Institute of Aquatic Biology, Chinese Academy of Sciences, cultured and amplified with BG11 culture medium. L. punctata (ZH0051), obtained from the Chengdu Institute of Biology, Chinese Academy of Sciences, was used with $1 / 5$ Hoagland medium for proliferation.

\subsection{Medium Formula and Culture Conditions}

\subsubsection{BG11 Culture Medium}

$\mathrm{NaNO}_{3} 1.5 \mathrm{~g} / \mathrm{L} ; \mathrm{K}_{2} \mathrm{HPO}_{4} 0.04 \mathrm{~g} / \mathrm{L} ; \mathrm{MgSO}_{4} \cdot 7 \mathrm{H}_{2} \mathrm{O} 0.075 \mathrm{~g} / \mathrm{L} ; \mathrm{CaCl}_{2} \cdot 2 \mathrm{H}_{2} \mathrm{O} 0.036 \mathrm{~g} / \mathrm{L} ;$ Citric acid $0.006 \mathrm{~g} / \mathrm{L}$; Ferric ammonium citric $0.006 \mathrm{~g} / \mathrm{L}$; EDTA $\cdot \mathrm{Na}_{2} 0.001 \mathrm{~g} / \mathrm{L} ; \mathrm{Na}_{2} \mathrm{CO}_{3} 0.02 \mathrm{~g} / \mathrm{L} ; \mathrm{A} 5: \mathrm{H}_{3} \mathrm{BO}_{3}$ $2.86 \mathrm{~g} / \mathrm{L}, \mathrm{MnCl}_{2} \cdot 4 \mathrm{H}_{2} \mathrm{O} 1.86 \mathrm{~g} / \mathrm{L}, \mathrm{ZnSO}_{4} \cdot 7 \mathrm{H}_{2} \mathrm{O} 0.22 \mathrm{~g} / \mathrm{L}, \mathrm{Na}_{2} \mathrm{MoO}_{4} \cdot 2 \mathrm{H}_{2} \mathrm{O} 0.39 \mathrm{~g} / \mathrm{L}, \mathrm{CuSO}_{4} \cdot 5 \mathrm{H}_{2} \mathrm{O} 0.08 \mathrm{~g} / \mathrm{L}$, $\mathrm{Co}\left(\mathrm{NO}_{3}\right)_{2} \cdot 6 \mathrm{H}_{2} \mathrm{O} 0.05 \mathrm{~g} / \mathrm{L}$. The $\mathrm{pH}$ value of the culture medium was adjusted to 6.5 , and reserved after sterilization. M. aeruginosa was inoculated in BG11 culture medium and culture in a light incubator with a temperature of $25^{\circ} \mathrm{C}$, light intensity of $2000 \mathrm{lux}$, and $16 \mathrm{~h} / 8 \mathrm{~h}$ photoperiod.

\subsubsection{Hoagland Culture Medium}

Stock Solution I: $\mathrm{Ca}\left(\mathrm{NO}_{3}\right)_{2} \cdot 4 \mathrm{H}_{2} \mathrm{O} 59 \mathrm{~g} / \mathrm{L}, \mathrm{KNO}_{3} 75.76 \mathrm{~g} / \mathrm{L}, \mathrm{KH}_{2} \mathrm{PO}_{4} 34 \mathrm{~g} / \mathrm{L}, \mathrm{HCl} 6 \mathrm{~mol} / \mathrm{L}$, $\mathrm{MgSO}_{4} \cdot 7 \mathrm{H}_{2} \mathrm{O} 50 \mathrm{~g} / \mathrm{L}$; Stock Solution II: $\mathrm{FeCl}_{3} \cdot 6 \mathrm{H}_{2} \mathrm{O} 5.4 \mathrm{~g} / \mathrm{L}$; Stock Solution III: EDTA $9 \mathrm{~g} / \mathrm{L}, \mathrm{KOH} 6 \mathrm{~mol} / \mathrm{L}$; Stock Solution IV: Tartaric acid $3 \mathrm{~g} / \mathrm{L}$; Stock Solution $\mathrm{V}_{2} \mathrm{H}_{3} \mathrm{BO}_{3} 2.86 \mathrm{~g} / \mathrm{L}, \mathrm{ZnSO}_{4} \cdot 7 \mathrm{H}_{2} \mathrm{O} 0.22 \mathrm{~g} / \mathrm{L}$, $\mathrm{Na}_{2} \mathrm{MoO}_{4} \cdot 2 \mathrm{H}_{2} \mathrm{O} 0.12 \mathrm{~g} / \mathrm{L}, \mathrm{CuSO}_{4} \cdot 5 \mathrm{H}_{2} \mathrm{O} 0.08 \mathrm{~g} / \mathrm{L}, \mathrm{MnCl}_{2} \cdot 4 \mathrm{H}_{2} \mathrm{O} 3.62 \mathrm{~g} / \mathrm{L}$. The prepared Hoagland culture solution was diluted with distilled water five times, and then reserved. L. punctata was inoculated in Hoagland culture medium and culture in a light incubator with a temperature of $23^{\circ} \mathrm{C}$, light intensity of 2000 lux, for $16 \mathrm{~h} / 8 \mathrm{~h}$.

\subsection{Experimental Design}

Hoagland medium was used for the laboratory experiment, and the average culture temperature was $23{ }^{\circ} \mathrm{C}$, along with a $16 \mathrm{~h} / 8 \mathrm{~h}$ photoperiod. The experiment was performed in $17.5 \mathrm{~cm} \times 12.5 \mathrm{~cm} \times 5.5 \mathrm{~cm}(1 \mathrm{~L})$ plastic containers, which were filled with $800 \mathrm{~mL}$ one fifth of Hoagland medium. M. aeruginosa was cultured in BG11 medium until the algae density reached $10^{7}$ cells $/ \mathrm{mL}$, then $M$. aeruginosa was inoculated into one fifth of Hoagland medium, with the final 
density being 0 cells $/ \mathrm{L}, 2 \times 10^{8}$ cells $/ \mathrm{L}, 4 \times 10^{8}$ cells $/ \mathrm{L}, 8 \times 10^{8}$ cells $/ \mathrm{L}$, and $1.6 \times 10^{9}$ cells $/ \mathrm{L}$, respectively. Except for the control group, the densities of M. aeruginosa all reached the "phytoplankton bloom" level, according to the classification criteria posed by [13]. L. punctata with good growth states were selected, cleaned with distilled water several times, then co-cultured with the above M. aeruginosa at a density of $200 \mathrm{mg} / \mathrm{m}^{2}$ (fresh weight). After 15 days of co-culture, the biomass, chlorophyll content, amylase activity, starch content, antioxidant enzyme activities, and malondialdehyde (MDA) content of L. punctata were determined. Besides, the MC content in both the culture medium and L. punctata were also determined. Of the Hoagland medium solution, 1/5 was supplemented during the whole experiment process, and each treatment included three replications. Each replication was measured three times, and the average value was taken.

\subsection{Experimental Methods}

\subsubsection{Determination of Growth Status of L. punctata}

The samples were harvested by collecting $75 \%$ of the container volume of L. punctata from the co-culture, followed by being rinsed with tap water many times, filled in nylon mesh bags, dehydrated for $5 \mathrm{~min}$ with a dehydrator, and dried on filter paper. The fresh weight of the sample was measured by using Bergmann's method [14]. The fresh weight growth rate $=$ final fresh weight (g)/initial fresh weight (g) [15].

\subsubsection{Screening Co-Culture Density of L. punctata and M. aeruginosa}

The fresh weight growth rate of L. punctata at inoculation densities of $100 \mathrm{~g} / \mathrm{m}^{2}, 200 \mathrm{~g} / \mathrm{m}^{2}, 400 \mathrm{~g} / \mathrm{m}^{2}$, and $800 \mathrm{~g} / \mathrm{m}^{2}$ was determined when cultured alone, and the fresh weight growth rate after co-culturing with a series of $M$. aeruginosa was also determined as the screening basis of experimental co-culture density. For dry weight determination, $5 \mathrm{~g}$ fresh-weight $L$. punctata was dried to constant weight in an oven at $50{ }^{\circ} \mathrm{C}$ and reweighed. The chlorophyll content was measured form $0.1 \mathrm{~g}$ fresh-weight L. punctata, based on the method mentioned by Arnon [16].

\subsubsection{Determination of Antioxidase Activities of L. punctata}

For the determination of antioxidase activities of L. punctata, $0.1 \mathrm{~g}$ of duckweed (fresh weight) was homogenized in $1 \mathrm{~mL}$ PBS on ice for extraction, the homogenate was centrifuged at $4{ }^{\circ} \mathrm{C}$ for $10 \mathrm{~min}$, and the supernatant sampled for measurement. The activities of ascorbate peroxidase (APX), catalase (CAT), peroxidase (POD), and superoxide dismutase (SOD) were detected, and malondialdehyde (MDA) content was measured following the instructions of the corresponding assay kit (Keming Biotechnology Co., Ltd., Suzhou, China). Amylase activity was determined by using 3,5-dinitrosalicylic acid colorimetry, and starch content was determined by anthrone colorimetry, referring to the instructions of the amylase kit and plant starch content kit (Keming Biotechnology Co., Ltd., Suzhou, China).

\subsubsection{Determination of MC Content}

The MC content was determined by using the double antibody sandwich method, referring to the instructions of the ELISA kit of the Shanghai Enzyme-linked Biological Company.

\subsubsection{Determination of Removal Rate of TN and TP}

The content of TN was determined by using ultraviolet spectrophotometry with alkaline potassium persulfate digestion, and determination of TP content was done by ammonium molybdate spectrophotometry.

\subsection{Statistical Analysis}

All data shown in the figures were the mean of three replicated independent determinations and were statistically analyzed using IBM SPSS Statistics 19.0 (IBM Inc., New York, NY, USA). 
The figures were plotted using Graphpad Prism 7 software (Graphpad Software Inc., San Diego, CA, USA).

\section{Results}

\subsection{Screening Co-Culture Density of L. punctata and M. aeruginosa}

Both L. punctata and M. aeruginosa are aquatic organisms, and they have overlapping ecological niches, so there is a certain competition between them when they grow in a limited water resource. A large number of experiments have shown that both biotic and abiotic stress can significantly affect the growth process of organisms and reduce the biomass of stressed organisms [17]. Based on the results of the fresh weight growth rate of L. punctata, we judged the growth states of L. punctata and screened the optimal density for co-cultivation.

After 15 days of culture alone, the fresh weight growth rate of each group of L. punctata was determined. The growth rate was highest at the inoculation density of $400 \mathrm{~g} / \mathrm{m}^{2}$ (Figure 1a). However, when the inoculation density reached up to $800 \mathrm{mg} / \mathrm{m}^{2}$, the L. punctata showed negative growth, indicating that the L. punctata was under self-stress, so this density was first excluded for co-culture. The result showed that the L. punctata inoculation densities of $200 \mathrm{mg} / \mathrm{m}^{2}$ and $400 \mathrm{mg} / \mathrm{m}^{2}$ were suitable to study the stress response of M. aeruginosa to L. punctata (Figure 1b). In addition, the water surface coverage rate of each L. punctata inoculation density is shown in Table 1, and given the adverse effects of water surface coverage on the photosynthesis of $M$. aeruginosa, we selected $200 \mathrm{mg} / \mathrm{m}^{2}$ of L. punctata (50\% water coverage rate) rather than $400 \mathrm{mg} / \mathrm{m}^{2}$ (100\% water coverage rate) as the initial inoculation density for subsequent experiments.

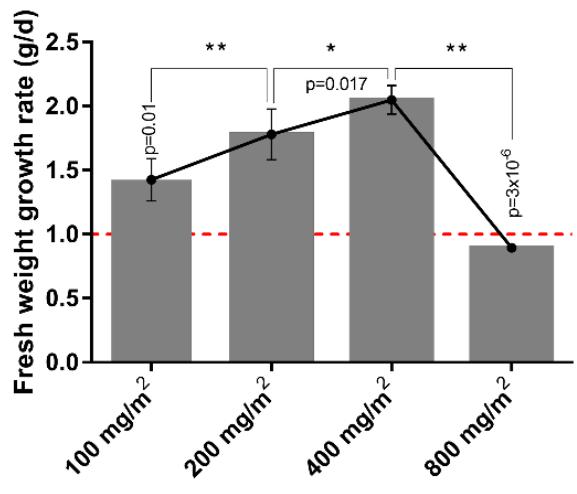

(a)

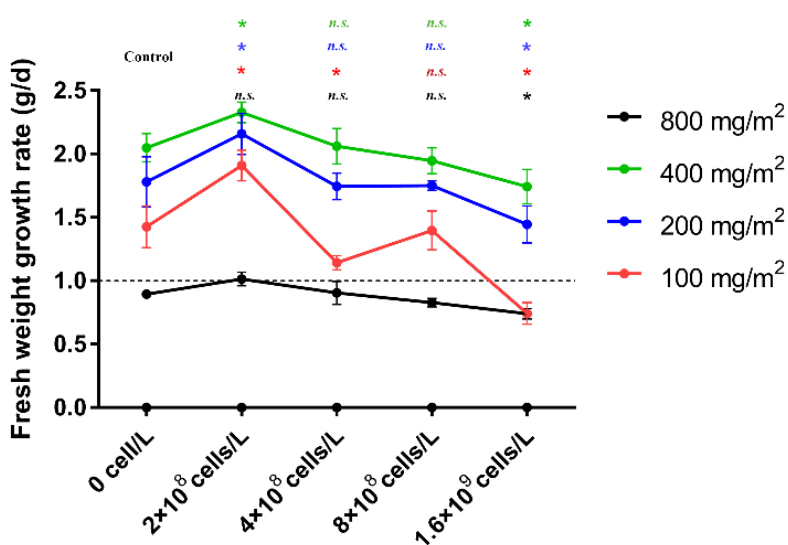

(b)

Figure 1. Fresh weight growth rates of L. punctata after 15 days of culture. (a) L. punctata cultured alone, and (b) L. punctata co-cultured with M. aeruginosa. Values are means $\pm \mathrm{SE}$ from three biological replicates. Asterisks indicate statistical significance based on one-way ANOVA; ${ }^{*} p<0.05,{ }^{* *} p<0.01$.

Table 1. The L. punctata water surface coverage rate of each inoculation density.

\begin{tabular}{ccccc}
\hline L. punctata density $\left(\mathrm{g} / \mathrm{m}^{2}\right)$ & 100 & 200 & 400 & 800 \\
\hline Water Surface Coverage Rate (\%) & 25 & 50 & 100 & 100 \\
\hline
\end{tabular}

\subsection{Effects of M. aeruginosa on the Growth of L. punctata}

M. aeruginosa had a significant inhibitory effect on the growth of L. punctata, and the inhibitory effect was dose-dependent. It is worth noting that the biomass of L. punctata, compared with the control, was significantly increased at $2 \times 10^{8}$ cells/L $M$. aeruginosa after 15 days of co-cultivation with $M$. aeruginosa (Figure 2a). In addition, the fresh weight of L. punctata decreased as the M. aeruginosa density increased in the culture medium. However, all the differences together did not reach a 
significant level compared with the control, except for the $1.6 \times 10^{9}$ treatment group. The change in trend of the dry weight was similar to that of fresh weight (Figure 2a).

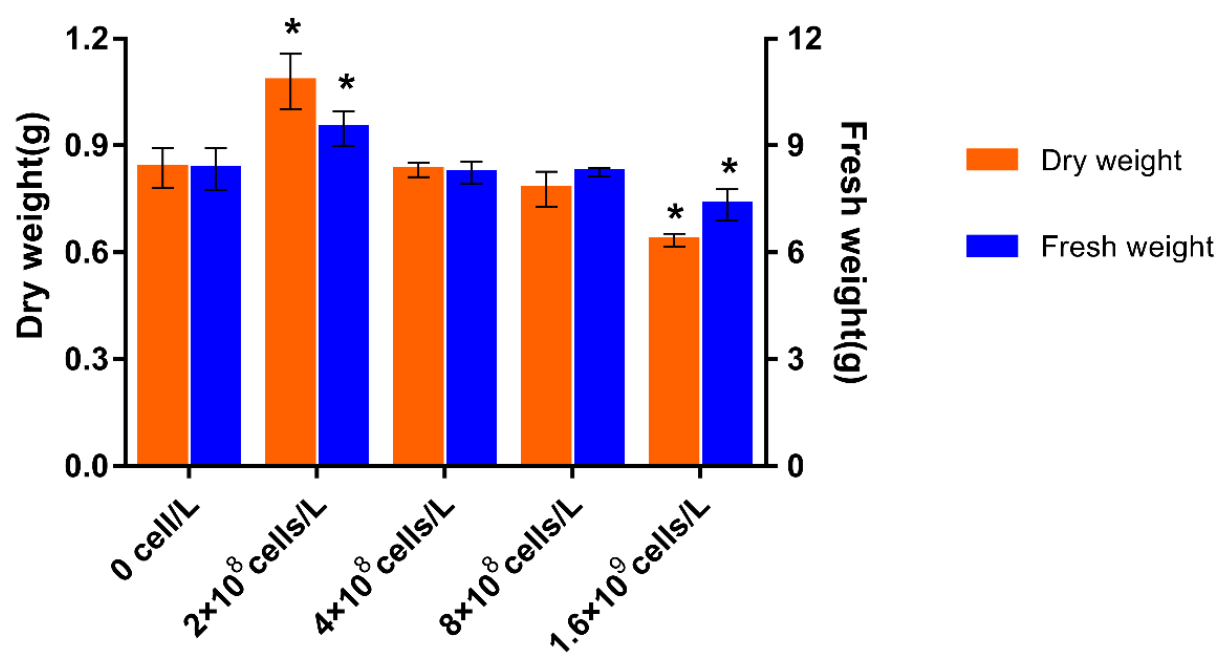

(a)

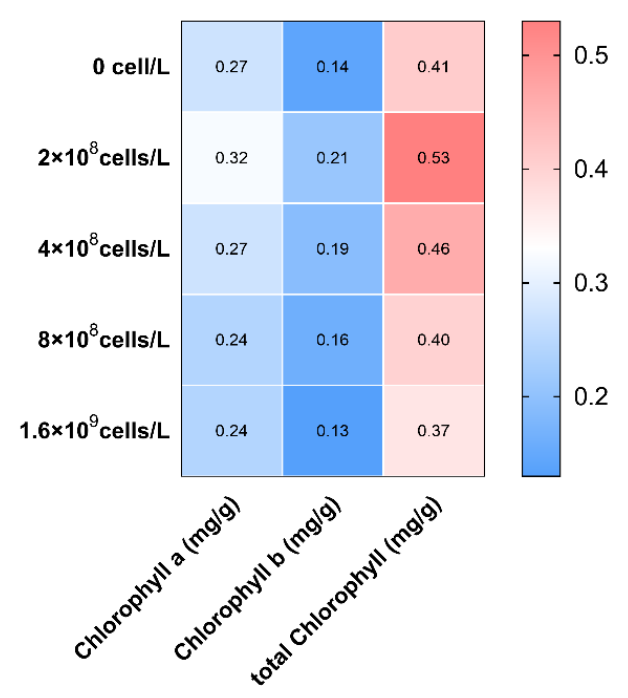

(b)

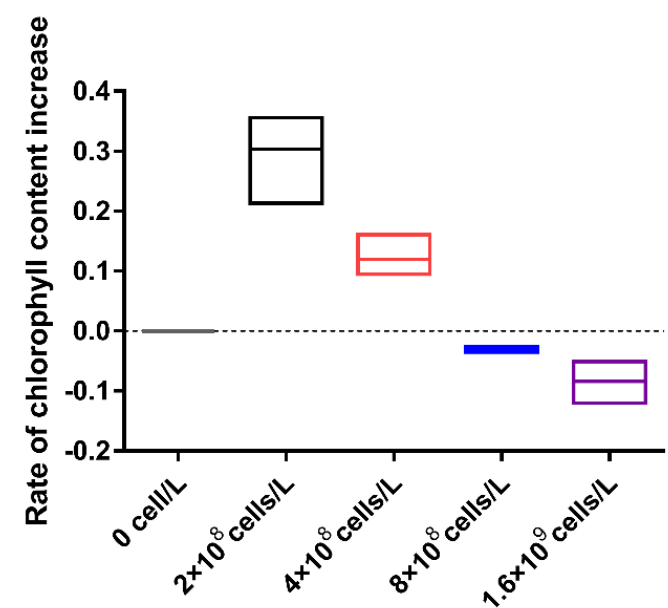

(c)

Figure 2. L. punctata growth states after co-culturing with M. aeruginosa. (a) Fresh biomass and dry biomass. (b) Chlorophyll content. (c) Rate of chlorophyll content increase. Values are means \pm SE from three biological replicates. Asterisks indicate statistical significance based on one-way ANOVA; * $p<0.05$.

Chlorophyll is the critical material basis for photosynthesis, and its content is the main indicator for judging the photosynthesis intensity of plants [18]. It can be seen from Figure $2 b$ that the change in trend of chlorophyll a and $\mathrm{b}$ content was basically consistent with the biomass. Compared with the control, the $2 \times 10^{8}$ cells/L $M$. aeruginosa treatment group was able to significantly increase the content of chlorophyll a and b in L. punctata. By contrast, the $1.6 \times 10^{9}$ cells/L M. aeruginosa treatment group significantly reduced the content of chlorophyll a and b (Figure 2b). Secondly, the effect of M. aeruginosa on chlorophyll a was more obvious than for chlorophyll b. The total chlorophyll content in L. punctata significantly increased from $0.41 \mathrm{mg} / \mathrm{g}$ to $0.53 \mathrm{mg} / \mathrm{g}$, and the increase rate reached $30.34 \%$ after treatment with $2 \times 10^{8}$ cells/L M. aeruginosa. The $1.6 \times 10^{9}$ cells/L M. aeruginosa was able to significantly reduce the total chlorophyll content of L. punctata by $8.37 \%$ compared with the control. 


\subsection{Effects of M. aeruginosa on Amylase and Starch Content of L. punctata}

Figure 3 a shows that the addition of $M$. aeruginosa had a significant effect on the $\alpha$ and $\beta$ amylase activity in L. punctata. Especially under the treatment of $2 \times 10^{8}$ cells/L M. aeruginosa, the activities of amylase $\alpha$ and $\beta$ increased significantly. With the increasing inoculation density of M. aeruginosa, the amylase activity of L. punctata in the corresponding co-culture system began to decrease, but it was still higher than the control. When the density of M. aeruginosa in the co-culture system became $4 \times 10^{8}$ cells/L, the enzyme activities of amylase $\alpha$ and $\beta$ in the L. punctata began getting lower than the control, and the activity of $\beta$-amylase decreased to a significant level. When the density of $M$. aeruginosa reached $1.6 \times 10^{9}$ cells/L, the activities of $\alpha$ and $\beta$ amylase decreased to the lowest level. As shown in Figure 3b, the result of starch accumulation in L. punctata is about $10.25 \mathrm{mg} / \mathrm{g}$ (fresh weight) under normal growth conditions. When the $M$. aeruginosa appeared in the culture system, the starch accumulation decreased significantly. With the continuous increase of the density of $M$. aeruginosa in the co-culture system, the starch accumulation in L. punctata gradually decreased. When the inoculation density of $M$. aeruginosa reached the highest level, the starch accumulation was as low as $7.51 \mathrm{mg} / \mathrm{g}$, and the starch accumulation decreased by $29.34 \%$.

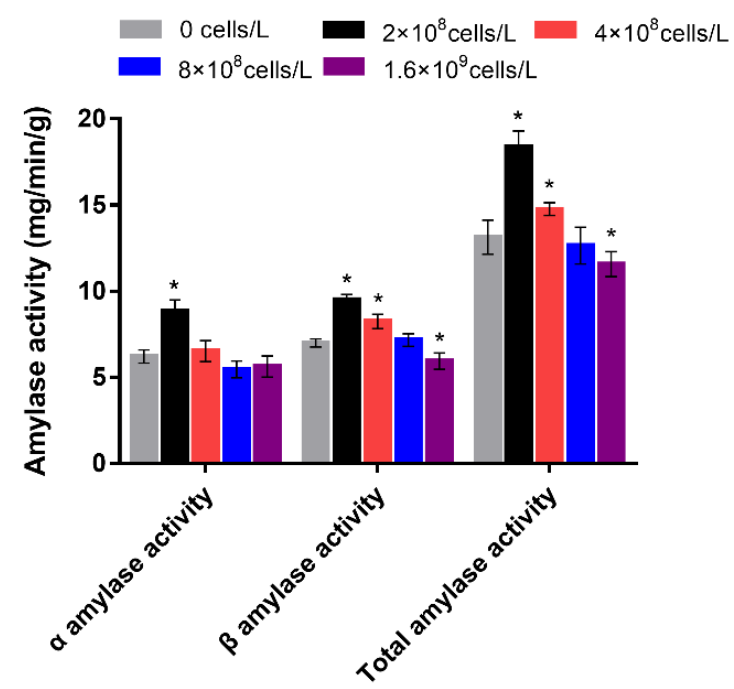

(a)

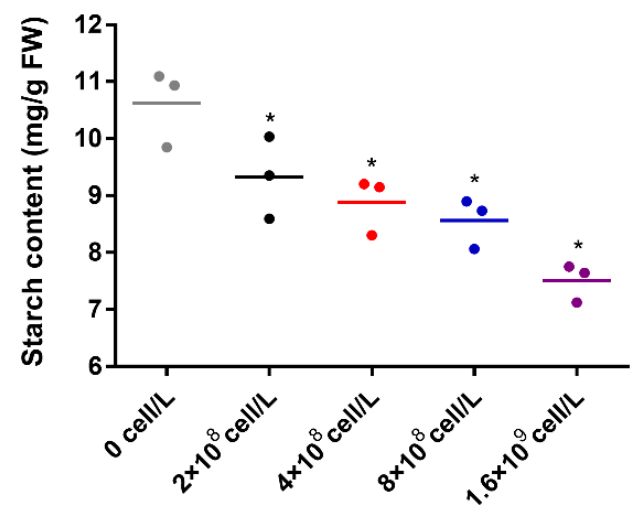

(b)

Figure 3. L. punctata amylase activity and starch content after co-culturing with M. aeruginosa. (a) $\alpha, \beta$, and total amylase activity. (b) Starch content. Values are means \pm SE from three biological replicates. Asterisks indicate statistical significance based on one-way ANOVA; ${ }^{*} p<0.05$.

\subsection{Effects of M. aeruginosa on Antioxidant System and MDA Accumulation of L. punctata}

The activities of four antioxidant enzymes in the antioxidant system of L. punctata co-cultured with $M$. aeruginosa was determined (Figure 4). The activities of ascorbate peroxidase (APX), catalase (CAT), peroxidase (POD), and superoxide dismutase (SOD) showed a trend of increasing first and then decreasing. The activities of APX, CAT, and POD increased significantly at the density of $2 \times 10^{8}$ cells/L of $M$. aeruginosa, and reached the peak values of $1.74 \mathrm{U} / \mathrm{g}, 248.19 \mathrm{U} / \mathrm{g}$, and $6066.67 \mathrm{U} / \mathrm{g}$, respectively. When the inoculation density of $M$. aeruginosa was $1.6 \times 10^{9}$ cells/L, the enzyme activity reduced to the lowest points of $0.83 \mathrm{U} / \mathrm{g}, 79.56 \mathrm{U} / \mathrm{g}$, and $3016.67 \mathrm{U} / \mathrm{g}$, respectively. SOD showed a relatively hysteretic response to $M$. aeruginosa, while there was no significant effect on its activity in treatment of $2 \times 10^{8}$ cells/L. Until the density of $M$. aeruginosa reached $4 \times 10^{8}$ cells/L, the activity of SOD reached its peak, $196.90 \mathrm{U} / \mathrm{g}$. At the highest density of M. aeruginosa, SOD activity decreased to the lowest value of $123.25 \mathrm{U} / \mathrm{g}$. 


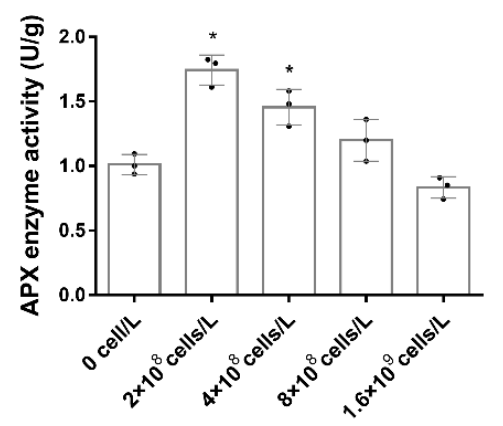

(a)

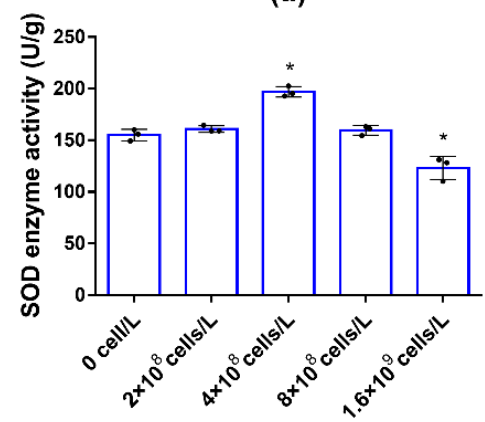

(d)

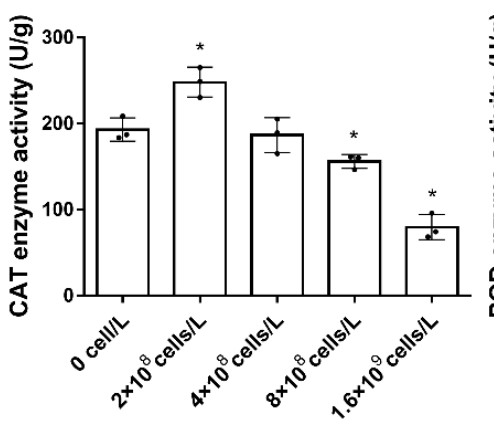

(b)

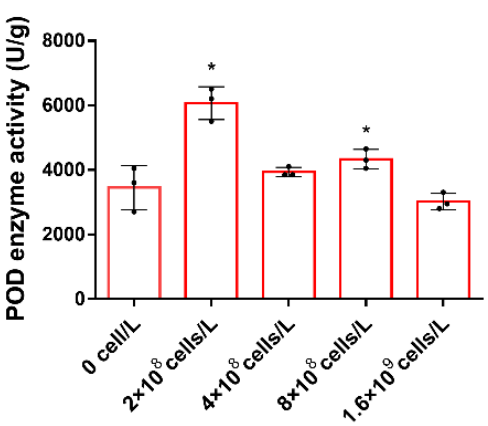

(c)

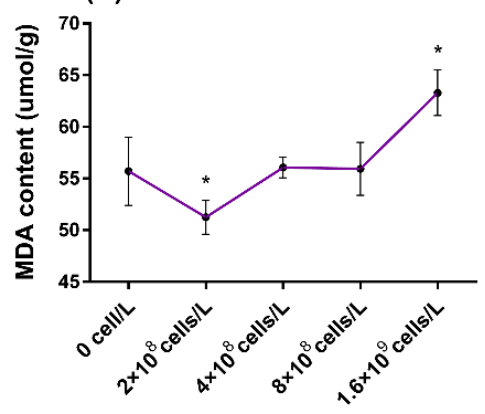

(e)

Figure 4. L. punctata APX (ascorbate peroxidase), CAT (catalase), POD (peroxidase), and SOD (superoxide dismutase) activity and MDA (malondialdehyde) content after co-culturing with M. aeruginosa. (a) APX activity. (b) CAT activity. (c) POD activity. (d) SOD activity. (e) MDA content. Values are means \pm SE from three biological replicates. Asterisks indicate statistical significance based on one-way ANOVA; ${ }^{*} p<0.05$.

In the $2 \times 10^{8}$ cells/L treatment, the L. punctata MDA content was significantly lower than the control (Figure 4e). With the increasing density of M. aeruginosa in the co-culture system, the content of MDA in the L. punctata also increased. When the inoculation density of M. aeruginosa was $1.6 \times 10^{9}$ cells $/ \mathrm{L}$, the content of MDA in L. punctata reached the highest value of $63.31 \mu \mathrm{mol} / \mathrm{g}$.

\subsection{Effect of Water Purification by L. punctata}

When M. aeruginosa was cultured alone, the content of MCs in the culture medium increased with the increase of $M$. aeruginosa. When the inoculation density was $1.6 \times 10^{9}$ cells/L, the content of MCs in the water reached the peak of $13.94 \mathrm{~g} / \mathrm{mL}$. However, when M. aeruginosa was co-cultured with L. punctata, the content of MCs in the culture medium was less than that of M. aeruginosa cultured alone (Figure 5a). In co-culturing, the removal rate of MCs by L. punctata showed a trend of first increasing and then decreasing with the increase of the density of M. aeruginosa, and the highest removal rate was $29.48 \%$ when the inoculation density of $M$. aeruginosa was $4 \times 10^{8}$ cells/L (Figure $5 \mathrm{~b}$ ).

The MCs absorbed by the L. punctata decreased gradually with the increase of the inoculation amount of $M$. aeruginosa. When the inoculation density was $2 \times 10^{8}$ cells/L, the content reached the peak value of $142.73 \mu \mathrm{g} / \mathrm{g}$. When the inoculum density of $M$. aeruginosa reached the highest point, MC accumulation in the L. punctata was reduced to a minimum of $114.84 \mu \mathrm{g} / \mathrm{g}$ (Figure $5 \mathrm{a}$ ). Meanwhile, the TN removal rate in the co-culture system was determined as follows: the TN removal rate increased with the increase of the density of $M$. aeruginosa, and when the density of $M$. aeruginosa was $8 \times 10^{8}$ cells/ $\mathrm{L}$, it reached the peak (36.94). When $200 \mathrm{mg} / \mathrm{m}^{2}$ of L. punctata was co-cultured in the culture system, the TN removal rate increased significantly, especially in the co-culture group of $2 \times 10^{8}$ cells/L M. aeruginosa, where the removal rate increased by $20.62 \%$. Then, with the increase of the density of $M$. aeruginosa, the TN removal rate gradually decreased. The trend of the TP removal rate was different with TN. In all the co-culture combinations, the TP removal rate was significantly higher than the single removal rate of M. aeruginosa (Figure 6). 

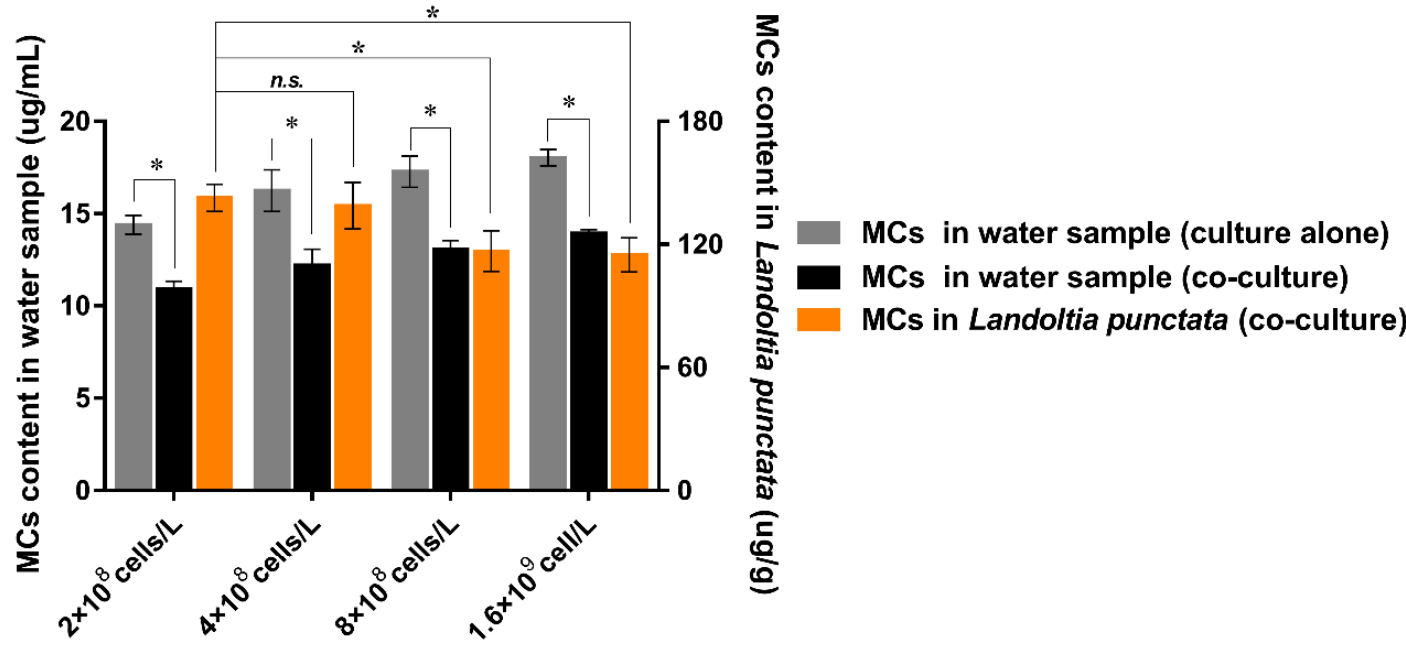

(a)

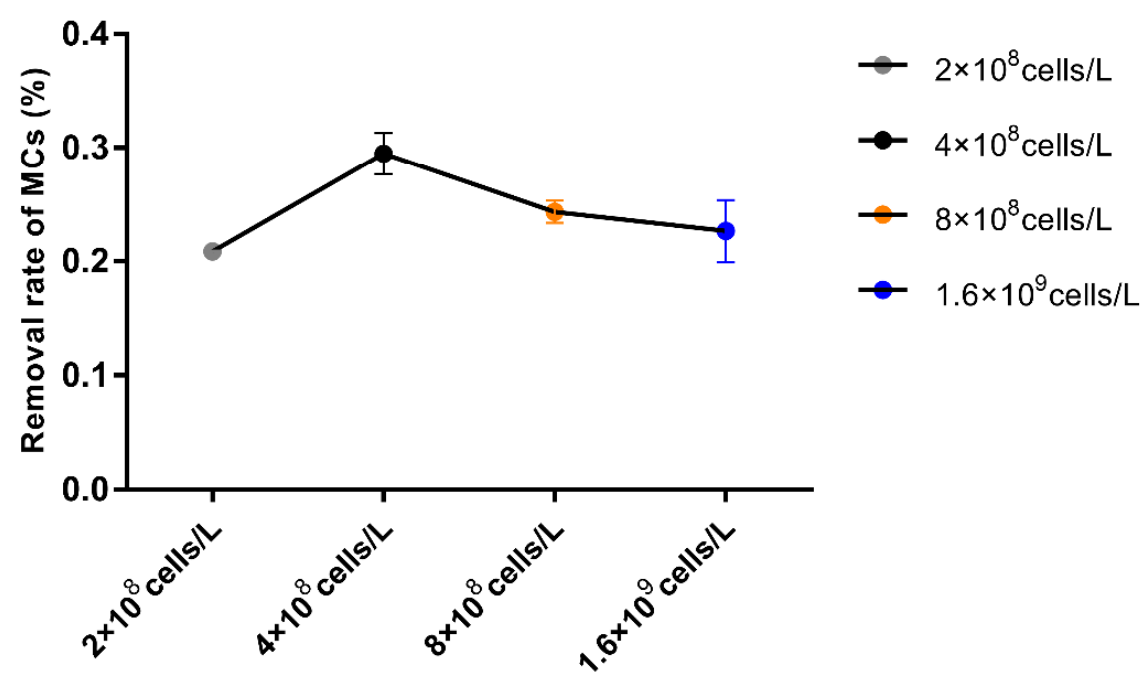

(b)

Figure 5. MC (microcystins) content and removal rate by L. punctata. (a) MC content in water sample and L. punctata. (b) Removal rate of MCs by L. punctata. Values are means \pm SE from three biological replicates. Asterisks indicate statistical significance based on Student's $t$ test; ${ }^{*} p<0.05$, n.s. indicates no significance. 
(a)

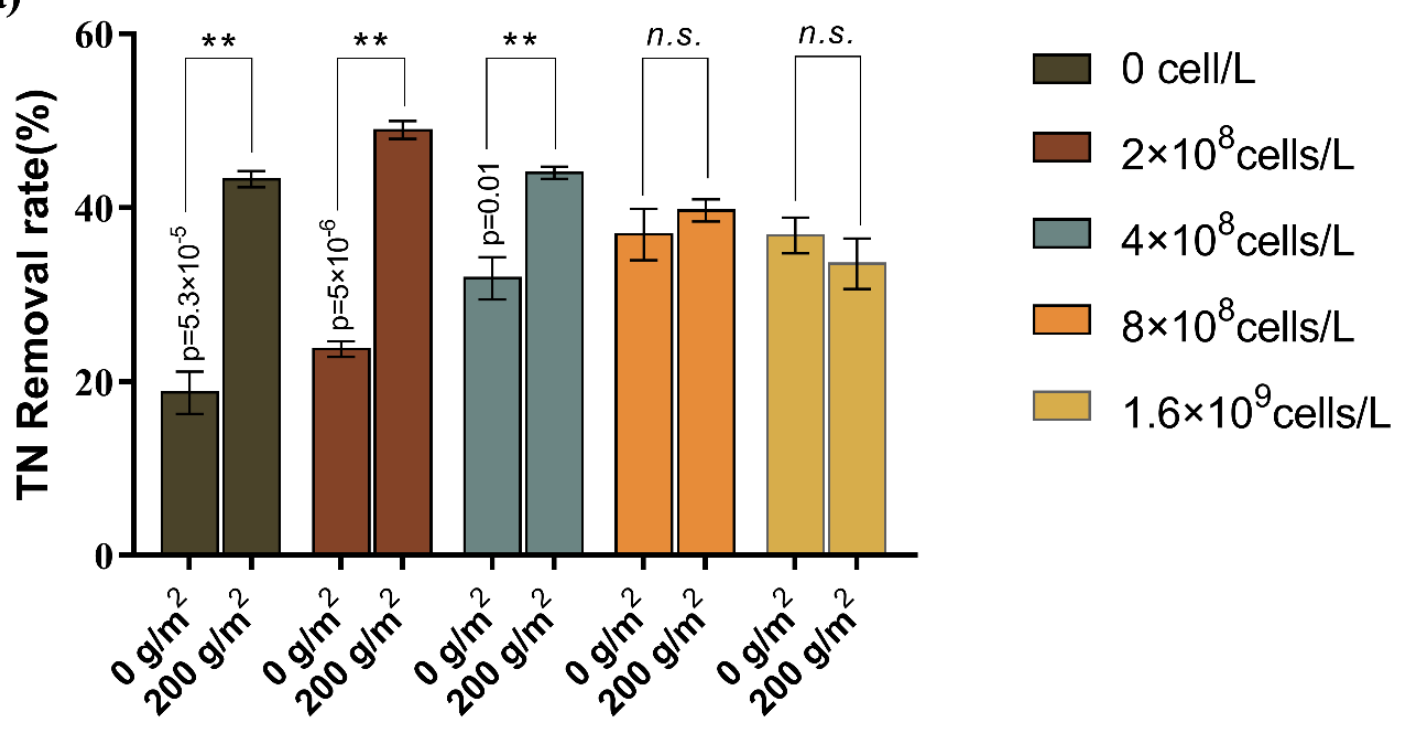

(b)

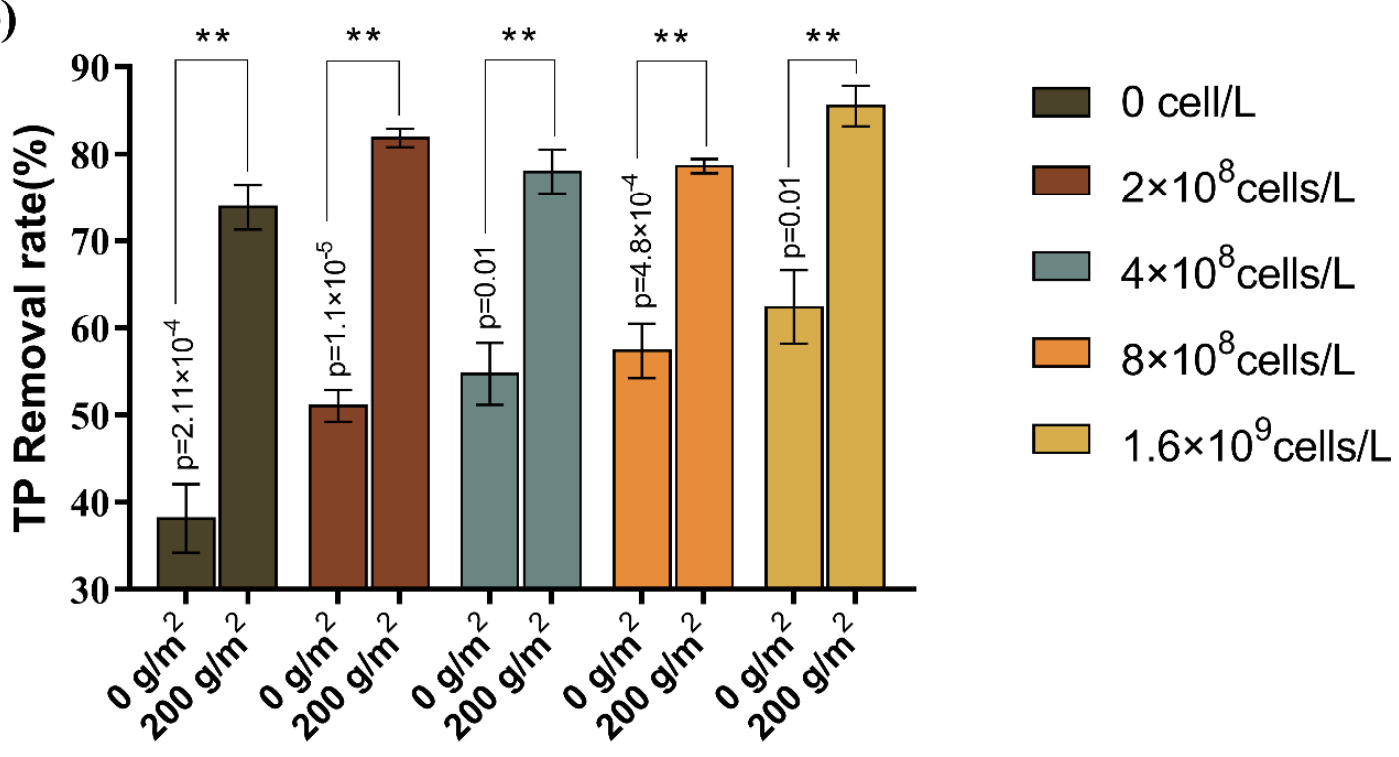

Figure 6. Total nitrogen (TN) and total phosphorus (TP) removal rate by L. punctata. (a) TN removal rate. (b) TP removal rate. Values are means \pm SE from three biological replicates. Asterisks indicate statistical significance based on Student's $t$ test; ${ }^{* *} p<0.01$, n.s. indicates no significance.

\section{Discussion}

\subsection{Effects of M. aeruginosa on the Growth of L. punctata}

Although Chen et al. [19] found that L. punctata has a good repair effect on eutrophic water, little is known about the changes in the physiological and biochemical indexes of L. punctata during the repair process. In this study, we found that after 15 days of co-cultivation of L. punctata and the gradient density M. aeruginosa was higher than the "water bloom" burst level, the biomass of L. punctata showed a trend of increasing first and then decreasing. When the number of M. aeruginosa was $2 \times 10^{8}$ cells $/ \mathrm{L}$ in the co-culture system, it was able to stimulate L. punctata to absorb nutrients to a 
certain extent. At the same time, the $\mathrm{CO}_{2}$ released by the respiration of $M$. aeruginosa could also increase the photosynthesis efficiency of L. punctata, so the density of M. aeruginosa promoted the growth of $L$. punctata. When the density of $M$. aeruginosa reached $1.6 \times 10^{9}$ cells/L, the stress of $M$. aeruginosa significantly inhibited the growth of L. punctata, and the fresh biomass and dry biomass of L. punctata decreased significantly compared with the control. Based on our results, we boldly conclude that during the process of increasing the density of $M$. aeruginosa in the co-culture system from $2 \times 10^{8}$ cells/ $\mathrm{L}$ to $1.6 \times 10^{9}$ cells/L, the L. punctata, under the stress of $M$. aeruginosa, experienced the following three stages: A beneficial period (co-culturing with $2 \times 10^{8}$ cells/L M. aeruginosa), ineffective stress period (co-culturing $4 \times 10^{8}$ cells/L-8 $\times 10^{8}$ cells/L M. aeruginosa), and effective stress period (co-culturing with $1.6 \times 10^{9}$ cells/L M. aeruginosa).

M. aeruginosa not only reduces nutrient uptake by duckweeds, but also changes the water environment and reduces dissolved oxygen in water when its biomass reaches a certain level, which causes bloom. Meanwhile, M. aeruginosa can also produce MCs, which causes stress on L. punctata, reduces its chlorophyll content, and inhibits its photosynthetic efficiency [20], affecting the growth of L. punctata. The determination of physiological and biochemical indexes of L. punctata in different periods can make the stress response of L. punctata to M. aeruginosa in the co-culture system be better understood.

\subsection{Effects of M. aeruginosa on Amylase and Starch Content of L. punctata}

The L. punctata activities of $\alpha$-amylase, $\beta$-amylase, and total amylase increased after co-culturing with $M$. aeruginosa at low density. $\alpha$-amylase is a kind of enzyme widely existing in plants, and relevant reports have proved that $\alpha$-amylase can be induced under certain stress conditions [21]. $\beta$-amylase is the key enzyme involved in starch hydrolysis in plants, and plays an indispensable role in coping with stress [22]. Studies have shown that starch hydrolysis into soluble sugar is an important way for plants to respond to stress $[23,24]$. More importantly, sugar itself can also act as a signaling molecule to regulate plant responses and growth under stress $[25,26]$. M. aeruginosa not only competes for nutrients with $L$. punctata, but also releases toxic substances into the water after cell breakage, which enhances the degree of stress on L. punctata, promotes initiation of the stress protection mechanism, and increases amylase activity. However, when the density of M. aeruginosa gradually increased, and the damage caused by M. aeruginosa was aggravated to the point of irreparable, it resulted in the decrease of amylase activity.

The accumulation of starch content is closely related to amylase activity. In this study, when the inoculation amount of $M$. aeruginosa was $2 \times 10^{8}$ cells/L, the activity of $\alpha$ and $\beta$ amylase increased significantly compared with the control. Both $\alpha$ and $\beta$ amylases are hydrolytic enzymes, and an increase in their activity leads to the decrease of starch accumulation. With the increase of the inoculation quantity of $M$. aeruginosa, which reduced the activity of $\alpha$ and $\beta$ amylase, it can be deduced that the activity of starch synthetase and the accumulation of starch content in L. punctata was also reduced and affected.

\subsection{Effects of M. aeruginosa on Antioxidant System and MDA Accumulation of L. punctata}

The co-culturing of $M$. aeruginosa not only has a competitive relationship of nutrients with L. punctata, but also releases microcystins (MCs) alone with cell disruption when it grows to a certain period, which causes stress to L. punctata. MCs can inhibit the activities of protein phosphatase 1 and $2 \mathrm{~A}$ in aquatic plants [21] and participate in a series of physiological and biochemical activities, such as the phosphorylation and dephosphorylation of organisms, resulting in homeostasis imbalance and various adverse reactions, such as the production of reactive oxygen species (ROS), which can cause toxic effects on plant cell membranes, and so forth. The antioxidant enzyme system in plants can effectively remove ROS and protect plants. Research has found that MCs can increase ROS in plants and inhibit the growth of aquatic plants [27]. In our experiment, the activities of POD, CAT, and APX gradually increased, and the content of MDA decreased at $2 \times 10^{8}$ cells/L of $M$. aeruginosa. 
When treated with $4 \times 10^{8}$ cells/L $1.6 \times 10^{9}$ cells/L, the activity of POD, CAT, and APX gradually decreased, and the content of MDA increased. It indicates that the addition of $2 \times 10^{8}$ cells/L of $M$. aeruginosa improved the enzymatic activity of L. punctata to resist an adverse environment. When $4 \times 10^{8}$ cells/L 1.6 $\times 10^{9}$ cells/L M. aeruginosa was added, the stress on L. punctata increased and exceeded its tolerance, resulting in the destruction of the antioxidant system.

\subsection{Effect of Water Purification by L. punctata}

When the cells of M. aeruginosa rupture, they release MCs and pollute the water. Due to the harmful effects of MCs on the natural environment and the human body, its elimination has always been a research hotspot. Many studies have shown that bioremediation is the most effective way to remove MCs. Approximately $90 \%$ of soluble MCs was degraded when treated with MC-degrading bacteria belonging to Pseudomonas. In this experiment, when M. aeruginosa was cultured alone, the content of MCs in the culture medium increased with the increase of the density of $M$. aeruginosa. However, L. punctata co-cultured with M. aeruginosa can significantly reduce the content of MCs in culture medium. The removal of MCs from L. punctata decreased gradually with the increase of MC content in the culture medium, indicating that the high density of MC stress exceeded the tolerance range of L. punctata. With an increasing density of dead M. aeruginosa, the MC content in water will be higher, which leads to the excessive content of MCs in L. punctata, and harvesting L. punctata at this time will be a good strategy for water purification. At the same time, the harvested L. punctata can be burned to destroy the structure of MCs and make it lose its toxicity. The burned residue can also be used as fertilizer to return to field. The high content of nitrogen and phosphorus in eutrophicated water is beneficial to the growth and reproduction of M. aeruginosa. We found that $200 \mathrm{~mL}$ of L. punctata can effectively reduce the content of nitrogen and phosphorus in water, especially the content of phosphorus, and improve environments which have been polluted by nitrogen and phosphorus.

\section{Conclusions}

In M. aeruginosa with inoculation densities of $2 \times 10^{8}$ cells $/ \mathrm{L}, 4 \times 10^{8}$ cells $/ \mathrm{L}$, and $8 \times 10^{8}$ cells $/ \mathrm{L}$, which are all much more abundant than in water bloom, our experiments have shown that L. punctata can grow normally. The chlorophyll content and biomass were not significantly lower than those of the control group, and the antioxidant enzyme system remained in a relatively normal state, while the removal rate of MCs fluctuated above and below $27 \%$, which had a good remediation effect on the MC-polluted water body. Therefore, we have found that remediation of water polluted by M. aeruginosa $\left(2 \times 10^{8}\right.$ cells $/ \mathrm{L}, 4 \times 10^{8}$ cells $/ \mathrm{L}$, and $8 \times 10^{8}$ cells $\left./ \mathrm{L}\right)$ with $200 \mathrm{~g} / \mathrm{m}^{2}$ L. punctata can achieve a better effect.

Author Contributions: S.L. (Shi Li) and S.L. (Sixiu Le) designed and prepared the manuscript. S.L. (Shi Li) performed most of the experiments and wrote the draft of manuscript. G.L. and M.L. contributed to data analyses. Y.Z. and R.W. took part in interpretation of the results and manuscript preparation. All authors have read and agreed to the published version of the manuscript.

Funding: This research was funded by the Chinese National key R \& D program, grant number 2018YFC1802605.

Conflicts of Interest: The authors declare no conflict of interest.

\section{References}

1. Sinha, E.; Michalak, A.M.; Balaji, V. Eutrophication will increase during the 21st century as a result of precipitation changes. Science 2017, 357, 405-408. [CrossRef] [PubMed]

2. Tanabe, Y.; Hodoki, Y.; Sano, T.; Tada, K.; Watanabe, M.M. Adaptation of the Freshwater Bloom-Forming Cyanobacterium Microcystis aeruginosa to Brackish Water Is Driven by Recent Horizontal Transfer of Sucrose Genes. Front. Microbiol. 2018, 9, 1150. [CrossRef] [PubMed]

3. Yeh, T.; Kao, C.M.; Chen, W.H. Integrated phytoremediation focused on microbial investigation. RSC Adv. 2018, 8, 4680-4685. [CrossRef] 
4. Bassuney, D.; Tawfik, A. Baffled duck weed pond system for treatment of agricultural drainage water containing pharmaceuticals. Int. J. Phytoremediat. 2017, 19, 774-780. [CrossRef] [PubMed]

5. Xu, Y.; Ma, S.; Huang, M.; Peng, M.; Bog, M.; Sree, K.S.; Appenroth, K.-J.; Zhang, J. Species distribution, genetic diversity and barcoding in the duckweed family (Lemnaceae). Hydrobiologia 2014, 743, 75-87. [CrossRef]

6. Gupta, C.; Prakash, D. Duckweed: An effective tool for phyto-remediation. Toxicol. Environ. Chem. 2013, 95, 1256-1266. [CrossRef]

7. Li, J.; Liu, Y.; Zhang, P.; Zeng, G.; Cai, X.; Liu, S.; Yin, Y.; Hu, X.; Hu, X.; Tan, X. Growth inhibition and oxidative damage of Microcystis aeruginosa induced by crude extract of Sagittaria trifolia tubers. J. Environ. Sci. 2016, 43, 40-47. [CrossRef]

8. Massey, I.Y.; Yang, F.; Ding, Z.; Yang, S.; Guo, J.; Tezi, C.; Al-Osman, M.; Kamegni, R.B.; Zeng, W. Exposure routes and health effects of microcystins on animals and humans: A mini-review. Toxicon 2018, 151, 156-162. [CrossRef]

9. Tsuji, K.; Watanuki, T.; Kondo, F.; Watanabe, M.F.; Suzuki, S.; Nakazawa, H.; Suzuki, M.; Uchida, H.; Harada, K.-I. Stability of microcystins from cyanobacteria-II. Effect of UV light on decomposition and isomerization. Toxicon 1995, 33, 1619-1631. [CrossRef]

10. Rastogi, R.P.; Sinha, R.P.; Incharoensakdi, A. The cyanotoxin-microcystins: Current overview. Rev. Environ. Sci. Bio/Technol. 2014, 13, 215-249. [CrossRef]

11. Wang, Y.; Cao, Y.; Li, H.; Gong, A.; Han, J.; Qian, Z.; Chao, W. Removal of MCs by Bi2O2CO3: Adsorption and the potential of photocatalytic degradation. Environ. Sci. Pollut. Res. 2018, 25, 11867-11874. [CrossRef] [PubMed]

12. Su, Y.; Mennerich, A.; Urban, B. Coupled nutrient removal and biomass production with mixed algal culture: Impact of biotic and abiotic factors. Bioresour. Technol. 2012, 118, 469-476. [CrossRef] [PubMed]

13. Ying, L.I.; Shi, Z.; Zhang, Y.X.; Zhao, Q.L.; Aijun, L.I.; Jin, Y.; Cheng, T. Evaluation Method and Application on Cyanobacteria Bloom Degree Classification With Algal Density. Environ. Sustain. Dev. 2014, 2, 67-68.

14. Chen, Q.; Jin, Y.; Zhang, G.; Fang, Y.; Xiao, Y.; Zhao, H. Improving Production of Bioethanol from Duckweed (Landoltia punctata) by Pectinase Pretreatment. Energies 2012, 5, 3019-3032. [CrossRef]

15. Silva, P.D.P.; De Nys, R.; Paul, N. Seasonal growth dynamics and resilience of the green tide alga Cladophora coelothrix in high-nutrient tropical aquaculture. Aquac. Environ. Interact. 2012, 2, 253-266. [CrossRef]

16. Arnon, D.I. Copper Enzymes in Isolated Chloroplasts. Polyphenoloxidase in Beta Vulgaris. Plant Physiol. 1949, 24, 1-15. [CrossRef]

17. Pavlović, I.; Pěnčík, A.; Novak, O.; Vujčić, V.; Brkanac, S.R.; Lepedus, H.; Strnad, M.; Sondi, B.S. Short-term salt stress in Brassica rapa seedlings causes alterations in auxin metabolism. Plant Physiol. Biochem. 2018, 125, 74-84. [CrossRef]

18. Lin, Y.; Chang, Q.R.; Liu, M.Y.; Wang, S. Estimation of chlorophyll content in rice at different growth stages based on hyperspectral in yellow river irrigation zone. Agric. Res. Arid Areas 2018, 2, 45-52.

19. Chen, G.; Fang, Y.; Huang, J.; Zhao, Y.; Li, Q.; Lai, F.; Xu, Y.; Tian, X.; He, K.; Jin, Y.; et al. Duckweed systems for eutrophic water purification through converting wastewater nutrients to high-starch biomass: Comparative evaluation of three different genera (Spirodela polyrhiza, Lemna minor and Landoltia punctata) in monoculture or polyculture. RSC Adv. 2018, 8, 17927-17937. [CrossRef]

20. Hu, Z.-Q.; Liu, Y.-D.; Li, D.; Dauta, A. Growth and antioxidant system of the cyanobacterium Synechococcus elongatus in response to microcystin-RR. Hydrobiologia 2005, 534, 23-29. [CrossRef]

21. Ziková, A.; Palíková, M.; Mareš, J.; Navrátil, S.; Kopp, R. Impacts of dietary cyanobacteria on fish. Acta Univ. Agric. Silvic. Mendel. Brun. 2010, 58, 277-284. [CrossRef]

22. Monroe, J.D.; Storm, A.R. Review: The Arabidopsis $\beta$-amylase (BAM) gene family: Diversity of form and function. Plant Sci. 2018, 276, 163-170. [CrossRef] [PubMed]

23. Lee, J.H.; Yu, D.J.; Kim, S.J.; Choi, I.; Lee, H.J. Intraspecies differences in cold hardiness, carbohydrate content and -amylase gene expression of Vaccinium corymbosum during cold acclimation and deacclimation. Tree Physiol. 2012, 32, 1533-1540. [CrossRef] [PubMed]

24. Benina, M.; Obata, T.; Mehterov, N.; Ivanov, I.; Petrov, V.; Toneva, V.; Fernie, A.R.; Gechev, T.S. Comparative metabolic profiling of Haberlea rhodopensis, Thellungiella halophyla, and Arabidopsis thaliana exposed to low temperature. Front. Plant Sci. 2013, 4, 499-510. [CrossRef] [PubMed] 
25. Smeekens, S.; Ma, J.; Hanson, J.; Rolland, F.A. Sugar signals and molecular networks controlling plant growth. Curr. Opin. Plant Boil. 2010, 13, 273-278. [CrossRef]

26. Meng, N.; He, M.; Bai, Y.; Xu, H.; Dandekar, A.M.; Fei, Z.; Cheng, L. Decreased sorbitol synthesis leads to abnormal stamen development and reduced pollen tube growth via an MYB transcription factor, MdMYB39L, in apple (Malus domestica). New Phytol. 2017, 217, 641-656. [CrossRef]

27. Ha, M.-H.; Pflugmacher, S. Time-dependent alterations in growth, photosynthetic pigments and enzymatic defense systems of submerged Ceratophyllum demersum during exposure to the cyanobacterial neurotoxin anatoxin-a. Aquat. Toxicol. 2013, 138, 26-34. [CrossRef] [PubMed]

(C) 2020 by the authors. Licensee MDPI, Basel, Switzerland. This article is an open access article distributed under the terms and conditions of the Creative Commons Attribution (CC BY) license (http://creativecommons.org/licenses/by/4.0/). 